Research report

\title{
BDNF-stimulated intracellular signalling mechanisms underlie exercise-induced improvement in spatial memory in the male Wistar
}

rat

\author{
Ranya G. Bechara ${ }^{a, b, *}$, Ronan Lyne ${ }^{a}$ Áine M. Kelly ${ }^{a, b}$ \\ a Trinity College Institute of Neuroscience, University of Dublin, Trinity College, Dublin 2, Ireland \\ ${ }^{\mathrm{b}}$ Department of Physiology, School of Medicine, University of Dublin, Trinity College, Dublin 2, Ireland
}

\section{H I G H L I G H T S}

- Exercised rats successfully completed challenging spatial task.

- Exercised rats showed increase in $\mathrm{KCl}$-stimulated BDNF release from dentate gyrus.

- Exercised rats displayed increases in BDNF expression and cell division in dentate gyrus.

- Exercised rats displayed activation of BDNF-stimulated signalling cascade.

- BDNF injections mimicked effects of exercise on both memory and signalling events.

\section{A R T I C L E I N F O}

\section{Article history:}

Received 18 August 2013

Received in revised form 6 November 2013

Accepted 11 November 2013

Available online $\mathrm{xxx}$

\section{Keywords:}

Brain-derived neurotrophic factor

Exercise

Spatial learning

Synaptic plasticity

Dentate gyrus

\begin{abstract}
A B S T R A C T
Exercise-induced improvements in learning are associated with neurotrophic and neurogenic changes in the dentate gyrus, but the intracellular signalling mechanisms that may mediate these improvements remain unknown. In the current study we investigate the effects of one week of forced exercise on spatial memory and analyse in parallel BDNF-stimulated signalling pathways in cells of the dentate gyrus. Additionally, we test whether a single intracerebroventricular (i.c.v.) injection of BDNF can mimic the observed cognitive and signalling changes. Male Wistar rats were assigned to exercised and sedentary groups and tested in a spatial task post-exercise. Tissue from the dentate gyrus was assessed for expression and release of BDNF, and for changes in expression and activation of TrkB, ERK and synapsin- 1 . In a separate set of experiments, male Wistar rats received a single i.c.v. injection of BDNF and were then tested in the same spatial learning task. Exercised and BDNF-treated (but not control) rats could successfully complete an object displacement task that tests spatial learning. Exercised rats and BDNF-treated rats displayed increases BDNF expression and ERK1 activation, while exercised rats showed increases in cell division, stimulated BDNF release, TrkB activation, and synapsin-1 expression in the dentate gyrus. We conclude that exercise-induced increases in BDNF in the dentate gyrus are sufficient to cause improvements in spatial memory by activating signalling cascades that enhance synaptic transmission in the hippocampus.
\end{abstract}

(ㄷ) 2013 Elsevier B.V. All rights reserved.

\section{Introduction}

Physical exercise is one of the most potent and effective cognitive enhancers [1]. Exercise can induce robust and consistent improvements in performance in different memory tasks in a variety of species [2]. Exercise has also been shown to boost neurogenic

\footnotetext{
* Corresponding author. Present address: Department of Pharmacology, University of Oxford, Mansfield Road, Oxford OX1 3QT, United Kingdom. Tel.: +44 07557552542; fax: +4401865 271853 .

E-mail addresses: becharar@tcd.ie, ranya.bechara@pharm.ox.ac.uk (R.G. Bechara).
}

[3] and neurotrophic [4] activity in the brain. Although substantial research now points to brain-derived neurotrophic factor (BDNF) as a central mediator of the positive effects of exercise on both cognition and neurogenesis [5,6], a causal role for BDNF in these exercise-induced changes has not yet been proven [7]. Furthermore, the specific mechanisms by which exercise increases BDNF expression in the brain and by which these increases translate into improvements in memory remain unclear. The role of BDNF in learning and memory has been well established [8-11]: BDNF has a wide range of short-term and long-term effects on neurons, most of which are thought to be mediated by its binding to the tyrosine kinase B (TrkB) Receptor, and the consequent activation of plasticity-related signalling cascades [12]. One such signalling

0166-4328/\$ - see front matter @ 2013 Elsevier B.V. All rights reserved. http://dx.doi.org/10.1016/j.bbr.2013.11.015 
cascade is the ERK (extracellular signal-related kinase) pathway [13], which has been shown to be essential to a number of forms of learning and memory $[14,15]$.

We have shown previously that one week of forced moderate exercise can improve memory in a challenging spatial task concomitant with an increase in BDNF expression in dentate gyrus, hippocampus and perirhinal cortex [16]. We have suggested that this increase in BDNF (and the subsequent stimulation of downstream signalling pathways) is directly responsible for the improvements observed in spatial and object recognition learning, via a mechanism that is distinct from that of environmental enrichment [17]. We have also shown that the intracerebroventricular administration of a physiological dose of exogenous BDNF is sufficient to mimic the improvement in object recognition learning induced by this exercise paradigm [16]. Here, we have extended this previous work to analyse the intracellular signalling mechanisms that may underlie the observed exercise-induced and BDNF-induced cognitive improvements. Specifically, we analysed the effects of exercise on the expression and release of BDNF and on the activation of the BDNF receptor, TrkB, and of ERK in the dentate gyrus. Since the BDNF-stimulated signalling cascades under investigation in this study have also been shown to be activated by learning, a group of naive cage controls was assessed in parallel to control for effects of learning on cell signalling.

Studies also suggest that exercise stimulates further changes in the hippocampus that could underlie memory improvements, notably an increase in the expression of synaptic vesicle proteins such as synapsin-1 [6], and in neurogenesis [18]. To monitor these changes, we also analysed cell division and synapsin- 1 expression in the dentate gyrus of these rats. Furthermore, we investigated whether a single intracerebroventricular (i.c.v.) injection of BDNF at a physiological dose can improve spatial memory and induce signalling events that facilitate synaptic transmission and plasticity, similar to those observed with exercise.

\section{Materials and methods}

\subsection{Animals}

In this study, we used three-month-old male Wistar rats (250-350 g) were obtained from the BioResources Unit, Trinity College Dublin. Rats were housed in groups of three, provided with food and water ad libitum and experienced a 12:12-h light-dark cycle in a temperature-controlled environment $\left(20-22^{\circ} \mathrm{C}\right)$. All experiments were conducted under national law and European Union directives on animal experiments.

\subsection{Exercise programme}

Rats were familiarised to motorised treadmills (Exer 3/6 treadmill, Columbus Instruments) by running on the treadmill for $15 \mathrm{~min}$ on 2 consecutive days (belt speed, $5-10 \mathrm{~m} / \mathrm{min}$ ), and then randomly assigned to an exercising group (Ex, $n=6$ ), a sedentary group (Sed, $n=6$ ). A forced exercise protocol was chosen to make it possible to control for the duration, intensity, and timing of exercise. The exercise protocol consisted of seven consecutive days of running for $1 \mathrm{~h}$ per day (belt speed $10-15 \mathrm{~m} / \mathrm{min}$ ). The treadmill is equipped with wire loops at one end of the belt through which a mild electric shock can be delivered. These act to motivate the rats to run continuously and were activated at low levels (on average an intensity of 2 on a scale of $0-10$; this represents a current of $0.7 \mathrm{~mA}$ with an interpulse interval of $2 \mathrm{~s}$ ) throughout all exercise sessions. Rats were observed while exercising to ensure they ran continuously and to monitor for signs of stress. Rats in the Sed groups were placed on stationary treadmills with shock loops activated at low levels for the same duration. Training in the object displacement task was undertaken on the final day of the exercise protocol.

\subsection{BrdU injections}

Animals received daily intra-peritoneal injections of BrdU $(50 \mathrm{mg} / \mathrm{kg}$ ) during the 7-day forced exercise period. BrdU was dissolved in $0.9 \% \mathrm{NaCl}$ at a concentration of $20 \mathrm{mg} / \mathrm{ml}$ and the solution was filter-sterilized before injection [3]. On each day, animals were injected $15 \mathrm{~min}$ prior to being placed on the treadmill.

\subsection{Object displacement task}

The object displacement (OD) task was used to assess spatial memory. A schematic illustrating the protocol used is shown in Fig. 2. The apparatus consisted of a black circular open field (diameter, $1 \mathrm{~m}$; height, $0.5 \mathrm{~m}$ ) placed in a dimly-lit room. Rats were handled daily for 1 week and habituated to the experimental apparatus, with 20 min of exploration in the absence of objects each day for 2 days before the experiment was performed. Objects were constructed from toy bricks and were fixed to the floor of the open field, $15 \mathrm{~cm}$ from the walls. Objects were cleaned thoroughly between trials to ensure the absence of olfactory cues. The criteria for exploration were strictly based on active exploration in which rats had to be in direct contact with the object. This included any body part of the rat (paws, tail, body, tail, whiskers) directly touching the object. The time spent in active exploration of each object and the total exploration time were recorded in seconds using stopwatches. In the training phase, three objects $(A, B$, and $C)$ were positioned in the open field and a spatial cue was fixed to the wall. On the training day, rats were allowed to explore the objects for a single 5 min trial. For the testing phase, object $\mathrm{C}$ was moved from its original position to a new position and rats were reintroduced into the arena $24 \mathrm{~h}$ post-training, for a single $5 \mathrm{~min}$ trial. The time (in seconds) spent exploring each object was expressed as a percentage of the total exploration time.

\subsection{Tissue preparation}

Rats were killed by decapitation immediately following completion of behavioural tasks and brains were removed and hemisected on ice. The left hemisphere was prepared for immunohistochemistry; the hemisphere was placed on a cork disk, covered with O.C.T compound (R.A. Lamb LTD, Sussex, UK), and quickly immersed in liquid nitrogen. Frozen hemispheres were then covered in aluminium foil and stored at $-80^{\circ} \mathrm{C}$ until sectioned. The right hemisphere was subdissected freehand on ice [19] and the dentate gyrus was divided into 3 equal pieces for further analysis. One piece was cross chopped for assessment of BDNF release (described below); the second piece was stored in RNALater ${ }^{\mathrm{TM}}$ (350 $\mu \mathrm{l}$; Ambion, Warrington, UK), for RNA extraction as described below; the third piece was immediately homogenised in lysis buffer (350 $\mu \mathrm{l}$; NP-40, 1\% (v/v); Tris ( $\mathrm{pH} \mathrm{8.0),} 20 \mathrm{mM}$; NaCl, $137 \mathrm{mM}$; glycerol, 10\% (v/v); ethylenediaminetetracetic acid (EDTA), $2 \mathrm{mM}$; activated sodium orthovanadate $\left(\mathrm{Na}_{3} \mathrm{VO}_{4}\right), 1 \mathrm{mM}$; aprotinin; leupeptin) using a $1 \mathrm{ml}$ glass homogeniser, for later analysis by ELISA, SDS-PAGE and Western immunoblotting. A group of naive cage controls $(n=6)$ were assessed in parallel for BDNF release, TrkB activation, ERK activation, and synapsin-1, to control for effects of learning on cell signalling.

\subsection{Surgical procedure}

Rats ( $n=12)$ were anaesthetised with isofluorane (4\% induction, $2 \%$ maintenance). A single hole was drilled in the skull over the left ventricle (coordinates, bregma, $0.9 \mathrm{~mm}$; midline, $1.3 \mathrm{~mm}$ ) 
and a cannula (Bilaney Consultants, Kent, UK) was lowered slowly into the ventricle to a depth of $3.6 \mathrm{~mm}$ below the brain surface. A guide cannula (Plastics 1, Bilaney Consultants) was held in place and fixed to the skull with dental cement (Prestige Dental Products, West Yorkshire, UK). The incision was closed with surgical staples (Promed, Ireland). Rats were given 7-10 days to recover before being tested.

\subsection{BDNF injection}

After a two-day habituation period to the open field, cannulated rats were randomly assigned to a BDNF group (BDNF, $n=6$ ) or a control group (CytC, $n=5)$. BDNF rats were infused with a single bolus of human recombinant $\mathrm{BDNF}$ ( $10 \mathrm{ng}$ in CSF, i.c.v.). Control rats received a similar injection of cytochrome $\mathrm{c}(10 \mathrm{ng}$ in CSF, i.c.v.). Cytochrome $\mathrm{c}$ was injected as it is a soluble small molecular weight protein that is inactive extracellularly [20]. The molecular weight is similar to that of BDNF, and it is often used as a control for injecting BDNF into the brain $[16,21,22]$. 30 min post-injection, all animals were trained in the object displacement task, and tested $24 \mathrm{~h}$ later. A separate set of cannulated animals (BDNF, $n=6$, CytC, $n=6$ ) were sacrificed 30 min post-injection for tissue analysis. Rats were killed by cervical dislocation and decapitation; brains were removed immediately and the dentate gyrus was dissected free on ice and divided into three equal pieces for further analysis, as described above.

\subsection{Tissue analysis}

\subsubsection{Cell division}

Frozen hemispheres were incubated at $-20^{\circ} \mathrm{C}$ for $30 \mathrm{~min}$ before sectioning was performed using a cryostat (Leica CM1900). $10 \mu \mathrm{m}$ sections were transferred onto prepared slides for light microscopy. Slides were coded and relabeled to ensure analysis was carried out blind. All sections were stored at $-20^{\circ} \mathrm{C}$ until stained. Quantification of proliferating cells in the dentate gyrus was performed using 3,3'-diaminobenzidine (DAB)-linked staining. Frozen sections were allowed to thaw to room temperature for $10 \mathrm{~min}$. Water-repellent circles were drawn around mounted tissue sections with a Pap pen (Invitrogen). Slides were fixed in pre-cooled 100\% methanol (Sigma-Aldrich) for $10 \mathrm{~min}$, and washed in PBS for $3 \times 3$ min. Slides were incubated in $2 \mathrm{~N} \mathrm{HCl}$ (Fluka, $75 \mu \mathrm{l}$ per section) for $30 \mathrm{~min}$ at $37^{\circ} \mathrm{C}$ to denature the DNA and allow the antibody to bind to the incorporated BrdU. Slides were washed in $0.1 \mathrm{M}$ Borate buffer $(0.1 \mathrm{M}$ sodium borate in deionised water, $\mathrm{pH} 8.5)$ for $2 \times 5 \mathrm{~min}$ to neutralise the sections and then washed in PBS for $3 \times 3 \mathrm{~min}$. Slides were incubated in $0.3 \% \mathrm{H}_{2} \mathrm{O}_{2}$ (Sigma, $75 \mu \mathrm{l}$ per section) for $20 \mathrm{~min}$ at $\mathrm{RT}$ to block endogenous peroxidase. Slides were then incubated in blocking buffer consisting of a 1:5 dilution of Normal Rabbit Serum (Vector Laboratories, UK) in 1\% BSA in PBS (75 $\mu$ l per section) for $1 \mathrm{~h}$ at room temperature to block non-specific binding sites. Primary rat anti-BrdU (AbCam) was diluted in blocking buffer to a concentration of $1: 75$. Slides were incubated in primary rat anti-BrdU $(75 \mu \mathrm{l}$ per section) overnight at $4{ }^{\circ} \mathrm{C}$, washed in PBS for $3 \times 3 \mathrm{~min}$, and incubated in secondary antibody (Rabbit anti-rat, AbCam, diluted 1:500 in blocking buffer) for $30 \mathrm{~min}$ at room temperature. Slides were washed in PBS for $3 \times 3 \mathrm{~min}$, and incubated in Vectastain $\mathrm{ABC}$ solution (Vector Laboratories, UK) for $30 \mathrm{~min}$ at $4{ }^{\circ} \mathrm{C}$ to amplify the signal.

DAB chromagen (Dako, Denmark), a colour reagent, was activated by adding $1 \mu \mathrm{l}$ of $30 \% \mathrm{H}_{2} \mathrm{O}_{2}$ to $1 \mathrm{ml}$ of DAB. $50 \mu \mathrm{l}$ of colour reagent was added to each section and colour development was observed under the microscope. The colour reaction was stopped by immersing the slides in PBS as soon as brown cells started to appear. Slides were counterstained using filtered haematoxylin (Sigma) for $4 \times 3 \mathrm{~min}$, and rinsed briefly in water between incubations. Slides were washed in distilled $\mathrm{H}_{2} \mathrm{O}$ for $3 \mathrm{~min}$. Slides were dehydrated by washing with increasing concentrations of ethanol (70\%, 90\%, and $100 \%$ ) and immersed in xylene for $1 \mathrm{~s}$. Slides were mounted with DPX mountant (Sigma) and a coverslip and left to dry before counting. Slides were examined using a Nikon light microscope. A total of three sections per rat, with six views in each section, were counted. Blue cells were identified as haematoxylin-stained (haematoxylin stains all nuclei). Brown cells were identified as BrdU-positive cells (DAB-chromagen stained). BrdU-positive cell quantities were expressed as a percentage of total cell count.

\subsubsection{BDNF release}

Samples of dentate gyrus tissue were sliced bi-directionally to a thickness of $350 \mu \mathrm{m}$ using a Mcllwain tissue chopper (Campden Instruments Ltd., UK), washed twice in ice-cold oxygenated Krebs solution $\left(\mathrm{NaCl}, 136 \mathrm{mM} ; \mathrm{KCl}, 2.54 \mathrm{mM}\right.$; $\mathrm{KH}_{2} \mathrm{PO}_{4}, 1.18 \mathrm{mM}$; $\mathrm{Mg}_{2} \mathrm{SO}_{4} \cdot 7 \mathrm{H}_{2} \mathrm{O}, 1.18 \mathrm{mM}$; $\mathrm{NaHCO}_{3}, 16 \mathrm{mM}$; Glucose, $10 \mathrm{mM} ; \mathrm{CaCl}_{2}$, $2 \mathrm{mM}$ ), before being washed twice and finally stored at $-80^{\circ} \mathrm{C}$ in Krebs solution containing DMSO (10\% (v/v) [23]). For analysis, slices were thawed rapidly, rinsed and suspended in $250 \mu \mathrm{l}$ oxygenated Krebs solution at $37^{\circ} \mathrm{C}$ for $10 \mathrm{~min}$. Slices were centrifuged at $1000 \times g$ for $3 \mathrm{~min}$ and the supernatant was removed for later assessment of BDNF by ELISA (Enzyme Linked Immunosorbent Assay). Following this, the slices were stimulated with $250 \mu 1 \mathrm{Krebs}$ containing $50 \mathrm{mM} \mathrm{KCl}$ and incubated at $37^{\circ} \mathrm{C}$ with $\mathrm{O}_{2}$ for $10 \mathrm{~min}$. Tissue was centrifuged at $1000 \times g$ for $3 \mathrm{~min}$ and the supernatant was removed for later assessment of BDNF by ELISA.

\subsubsection{Analysis of BDNF and $p$-TrkB by ELISA}

The concentration of BDNF in samples generated from the BDNF release experiment was quantified by enzyme-linked immunosorbent assay (ELISA; R\&D Systems) according to the manufacturer's instructions. Briefly, 96-well plates (MaxiSorp; NUNC) were coated overnight at room temperature with anti-BDNF antibody $(100 \mu \mathrm{l}$; diluted $1: 180$ in phosphate buffered saline (PBS) $(137 \mathrm{mM} \mathrm{NaCl}$, $2.7 \mathrm{mM} \mathrm{KCl}, 8.1 \mathrm{mM} \mathrm{Na}_{2} \mathrm{HPO}_{4}, 1.5 \mathrm{mM} \mathrm{KH}_{2} \mathrm{PO}_{4} ; \mathrm{pH} 7.2$ ). The plates were washed three times in PBS-T ( $300 \mu \mathrm{l}$; 0.05\%Tween-20 in PBS) using an automated plate washer and blocked with block buffer $(300 \mu \mathrm{l}$; bovine serum albumin (BSA), $1 \%(\mathrm{v} / \mathrm{v}))$ for $1 \mathrm{~h}$ at room temperature. The plates were subsequently incubated with samples and serially-diluted BDNF standards $(50 \mu \mathrm{l})$ for $2 \mathrm{~h}$ at room temperature, washed, and incubated with anti-BDNF detection antibody ( $100 \mu \mathrm{l}$; diluted 1:180 in block buffer) for $2 \mathrm{~h}$ at room temperature. Plates were washed, incubated with Streptavidin Horse-Radish Peroxidase (HRP)-conjugated antibody $(100 \mu \mathrm{l})$ for $20 \mathrm{~min}$ at room temperature and washed again. TMB One solution $(100 \mu \mathrm{l})$ was added for $20 \mathrm{~min}$, the reaction was stopped with $1 \mathrm{~N} \mathrm{H}_{2} \mathrm{SO}_{4}(50 \mu \mathrm{l})$, and the absorbance was measured at $450 \mathrm{~nm}$ using a 96-well plate reader (Labsystems, Multiskan RC). Concentration of BDNF in samples was calculated by extrapolation from the standard curve, protein content was assessed by the method of Bradford [24] and results were expressed as pg BDNF/mg protein.

The concentration of p-TrkB in homogenate prepared from dentate gyrus was quantified by enzyme-linked immunosorbent assay (ELISA; R\&D Systems) according to the manufacturer's instructions. Briefly, a 96-well plate (NUNC-immuno ${ }^{\mathrm{TM}}$ MaxiSorp ${ }^{\mathrm{TM}}$ plate; Denmark) was coated with capture antibody $(2 \mu \mathrm{g} / \mathrm{ml}$ mouse anti-human p-TrkB antibody diluted in phosphate buffered saline $(50 \mu \mathrm{l} /$ well $)$ ) and incubated overnight at room temperature. The plate was washed with wash buffer (PBS-T; 0.05\% Tween ${ }^{\circledR} 20$ in PBS) using an automated plate washer (Columbus Plus, Tecan, Austria) and blocked with reagent diluent (1\% BSA in PBS; $150 \mu \mathrm{l} /$ well) for $1 \mathrm{~h}$ at room temperature. After washing, standards (recombinant human phosphorylated TrkB) were diluted in the recommended IC diluent (1\% NP-40, $20 \mathrm{mM}$ Tris ( $\mathrm{pH} 8.0$ ), $137 \mathrm{mM} \mathrm{NaCl}, 10 \%$ glycerol, $2 \mathrm{mM}$ EDTA, $1 \mathrm{mM}$ activated sodium orthovanadate) to 
a concentration of $300 \mathrm{pg} / \mathrm{ml}$ (top standard). The standard curve consisted of eight 1:2 serial dilutions, with the ninth standard containing only the IC diluent. Standards and samples (equalised in lysis Buffer) were added in duplicate $(50 \mu \mathrm{l} /$ well) and incubated for $2 \mathrm{~h}$ at room temperature. The plate was washed again and incubated with detection antibody ( $50 \mathrm{ng} / \mathrm{ml}$ mouse anti-phosphotyrosine antibody conjugated to HRP in IC diluent; $50 \mu \mathrm{l} /$ well) for $2 \mathrm{~h}$ at room temperature. After washing, either Substrate solution (1:1 mixture of colour reagent $A$ and colour reagent $B$ ) or TMB one solution was added $(50 \mu \mathrm{l} /$ well $)$ and incubated in the dark at room temperature for $20 \mathrm{~min}$. The reaction was stopped using $1 \mathrm{M}$ $\mathrm{H}_{2} \mathrm{SO}_{4}(50 \mu \mathrm{l} /$ well). The OD was read at $450 \mathrm{~nm}$ wavelength using a 96-well plate reader (Synergy HT Multi-Mode Microplate Reader, BioTek $\left.^{\circledR}\right)$. The regression equation of the standard curve was used to calculate the p-TrkB concentrations of the tissue homogenate, and protein concentrations of samples in lysis buffer were quantified using the BCA protein assay method (Pierce ${ }^{\circledR}$, Thermo Scientific). Results were expressed as pg p-TrkB/mg protein.

\subsubsection{Polymerase chain reaction (PCR)}

RNA extraction was performed on samples of dentate gyrus from the Sed $(n=6)$ and Ex $(n=6)$ groups using a total RNA isolation kit (Macherney-Nagel). Spectrophotometric measurements were carried out using the Nanodrop Spectrophotometer (Nanodrop Technologies Wilmington, DE, USA) to determine RNA concentration and purity. RNA samples were equalised in Sigma water. A high capacity cDNA archive kit (Applied Biosystems) was used to reverse transcribe the equalised RNA samples. Quantitative real-time PCR (qPCR) was performed with $50 \mathrm{ng}$ cDNA using custom-designed gene-expression assays for BDNF and TrkB. Gene expression of targets was assessed using Taqman gene expression assays (Applied Biosystems, UK) containing specific target primers, and FAM-labelled MGB target probes. $\beta$-Actin gene expression was used to normalise gene expression between samples, and was quantified using a $\beta$-actin endogenous control gene expression assay containing specific primers, and a VIC-labelled MGB probe for rat $\beta$-actin. Analysis was performed using the $\Delta \Delta C T$ method. Data are presented as mean relative quotient (RQ) values that represent fold changes relative to the mean value for controls.

\subsubsection{SDS PAGE and Western immunoblotting}

Protein concentration of samples prepared in Lysis Buffer was quantified using the BCA protein assay method (Pierce ${ }^{\circledR}$, Thermo Scientific). Samples were equalised in lysis buffer and $100 \mu \mathrm{l}$ of sample was added to $100 \mu \mathrm{l}$ of sample buffer (Tris- $\mathrm{HCl} \mathrm{pH}$ 6.8, 0.5 M; sodium dodecyl sulphate (SDS), $10 \%(\mathrm{w} / \mathrm{v})$; glycerol, $10 \%(\mathrm{v} / \mathrm{v}) ; 2-\beta$-mercaptoethanol, $5 \%(\mathrm{w} / \mathrm{v})$; bromophenol blue, $0.05 \%(\mathrm{w} / \mathrm{v})$ ). Samples were boiled in a heating block for $5 \mathrm{~min}$. $10 \%$ acrylamide gels were prepared and inserted into an electrophoresis unit (Bio-Rad Mini-Protean 3, Bio-Rad Laboratories, Herfortshire, England). Electrode running buffer (composition: Tris base, $25 \mathrm{mM}$; glycine, $200 \mathrm{mM}$; SDS, $17 \mathrm{mM}$ ) was added to the inner and outer reservoirs. $10 \mu \mathrm{l}$ of prepared sample or $5 \mu \mathrm{l}$ of prestained molecular markers (Bio-Rad Laboratories, USA) were loaded onto the wells. The gel was run at $30 \mathrm{~mA}$ for $30 \mathrm{~min}$ and washed in transfer buffer (composition: Tris base, $25 \mathrm{mM}$; glycine, $192 \mathrm{mM}$; methanol, 20\% (v/v); SDS, 0.5\% (w/v)). One sheet of nitrocellulose paper (Amersham) and two sheets of filter paper (Whatman No. 3) were pre-cut to the size of the gel and soaked in transfer buffer for $5 \mathrm{~min}$. A layered sandwich was prepared by stacking the nitrocellulose paper on top of the filter paper followed by the gel and the second piece of filter paper. Air bubbles were removed and the sandwich was placed on the anode of a semi-dry blotter (Apollo Instruments, Alpha Technologies, Dublin, Ireland) pre-moistened with transfer buffer. The transfer was carried out at $225 \mathrm{~mA}$ for $75 \mathrm{~min}$. Blots were blocked in blocking buffer (5\% BSA in TBS-T) for $2 \mathrm{~h}$ at room temperature, and probed with the appropriate primary antibody overnight (diluted in $2 \%$ BSA in TBS-T; p-ERK (1:1000), ERK (1:1000, all Cell Signaling Technology), or $\beta$-actin (1:1000; Sigma, UK). The primary antibody was washed off $(6 \times 10 \mathrm{~min}$ in TBS-T) and the blot was incubated with a secondary antibody for $1 \mathrm{~h}$ (Goat ant-rabbit, Sigma, diluted in $2 \%$ BSA in TBS-T to a concentration of $1: 1000$ ). The secondary antibody was washed off ( $6 \times 10 \mathrm{~min}$ in TBS-T). Immunoreactive bands were detected using SuperSignal West Dura chemiluminescence reagents (Pierce). The membranes were developed using a gel reader (Fujifilm Las-3000 Imaging System) and densitometry was performed using the Fujifilm Multigauge Software.

\subsection{Statistical analysis}

All data are presented as mean \pm S.E.M. Statistical analysis was performed in GraphPad Prism. For experiments with two groups and one independent variable a Student's t-test was used. For more than two groups and one independent variable, a one-way ANOVA with Post hoc Tukey comparisons was performed. A value of $p<0.05$ (*) was considered to be significant.

\section{Results}

\subsection{Effect of exercise on spatial learning}

The effect of one week of exercise on spatial memory was tested in an object displacement paradigm with three objects and 5 min of training (Fig. 1). Time spent exploring each of the objects was recorded and reported as a percentage of the total exploration time (mean $\pm S E M)$. On the training day, both groups of rats spent a similar amount of time exploring the three objects, indicating that there was no preference for any of the three objects (Fig. 1a). On the testing day, the sedentary group did not show preferential exploration of the displaced object, indicating that these rats were unable to learn the task. However, the exercised group spent significantly more time exploring the displaced object (C) compared to object A and Object B (Fig. 1b, $p=0.0013$, $F_{(2,15)}=10.74$; ANOVA), indicating that these rats were able to learn the task.

\subsection{Effect of exercise on BDNF expression and cell division in the dentate gyrus}

BDNF mRNA expression in the dentate gyrus was assessed by PCR. One week of forced exercise resulted in a significant increase in BDNF mRNA expression in the dentate gyrus compared to sedentary controls (Fig. 2a, $p<0.05$, Student's $t$-test). Cell division was assessed by analyzing the number of BrdU-positive cells using immunohistochemistry. The average number of BrdU-positive cells (as a percentage of the total number of cells counted) was significantly increased in the dentate gyrus of exercised rats compared to sedentary controls (Fig. $2 \mathrm{~b}-\mathrm{d} ; \mathrm{p}=0.0169$, Student's $t$-test).

\subsection{Effect of exercise on $\mathrm{KCl}$-stimulated release of BDNF from the dentate gyrus}

$\mathrm{KCl}$-stimulated BDNF release was assessed using an ELISA. A naïve control group $(n=6)$ was assessed in parallel to control for the possible effects of learning on BDNF release. Under constitutive conditions, no difference was observed in the amount of BDNF released from slices of dentate gyrus in any group (Fig. 3). Upon stimulation with $50 \mathrm{mM} \mathrm{KCl,} \mathrm{BDNF}$ release from the dentate gyrus 
a)
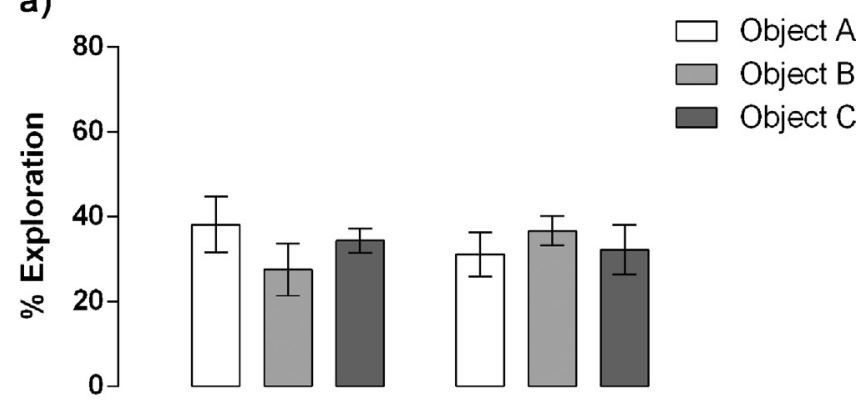

$\mp$

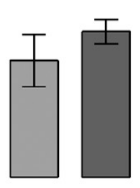

Sed

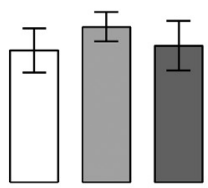

Ex

b)

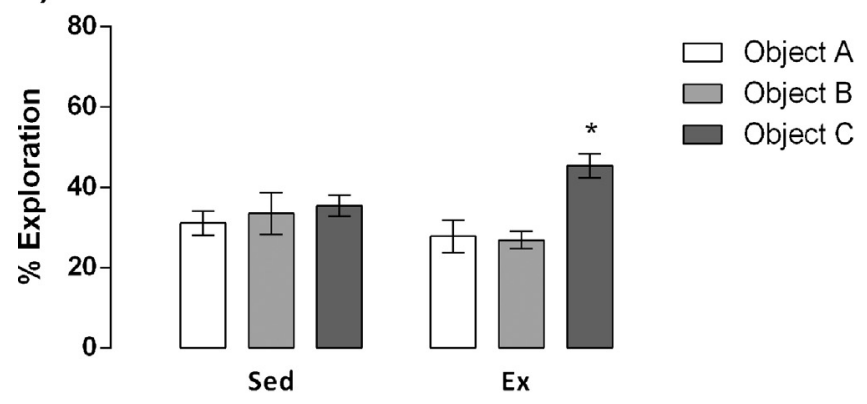

c)
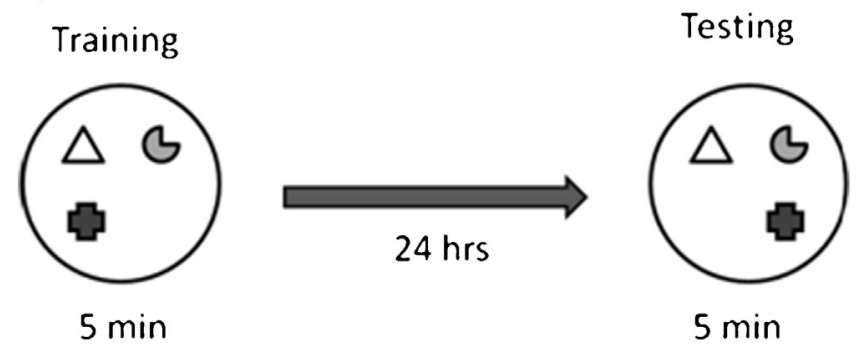

Fig. 1. Exercised rats display enhanced spatial learning. Data are presented as exploration time for each object ( $A, B$ or $C$, where $C$ is the displaced object) as a percentage of the total exploration time. Neither sedentary rats (Sed, $n=6$ ) nor exercised rats $(\mathrm{Ex}, n=6)$ preferentially explored any object on the training day (a). On the testing day, the exercised rats preferentially explored the displaced object, while the sedentary rats did not (c; * $p<0.05$; one-way ANOVA with Post hoc Tukey comparison). Data are presented as mean $\%$ exploration time for each object \pm SEM.

of the exercised group was significantly increased when compared with the naive group $\left(p<0.05, F_{(2,12)}=4.82\right.$, ANOVA).

\subsection{Effect of exercise on TrkB, ERK, and synapsin in the dentate gyrus}

Homogenate prepared from the dentate gyrus was analysed for TrkB activation by ELISA. There was a significant increase in phosphorylated TrkB in the dentate gyrus of exercised rats compared with naive controls (Fig. 4a, $p=0.03$, ANOVA). Homogenate prepared from the dentate gyrus was analysed for ERK activation and synapsin-1 expression by Western Blot analysis. Exercise induced a significant increase in phosphorylated ERK1 ( $\mathrm{p} 44$-ERK) compared with the naive group (Fig. 4b, $p=0.049$; ANOVA), when normalised to total ERK1. No changes were observed in p-ERK2 when normalised to total ERK2 (p42-ERK). No changes were observed in expression of total ERK1 or total ERK2. Western blot analysis revealed a significant increase in synapsin-1 in dentate gyrus of the exercised group when compared with the naive group (Fig. 4c, $p=0.041 ;$ ANOVA).

\subsection{Rats infused with BDNF (i.c.v.) display enhanced spatial learning}

The effect of BDNF infusions on spatial memory was tested in an object displacement paradigm with three objects and 5 min of training in an identical task to that used in the previous exercise experiment. On the training day, both groups of rats spent a similar amount of time exploring the three objects, indicating that there was no preference for the three objects (Fig. 5a). On the testing day, statistical analysis showed that the control group did not show preferential exploration of the displaced object, indicating that these rats were unable to learn the task. However, the BDNF group spent significantly more time exploring the displaced object $(C)$ compared to object $A$ and Object $B\left(p=0.0132, F_{(2,15)}=6.335\right.$; ANOVA), indicating that these rats were able to learn the task (Fig. 5b).

\subsection{A single BDNF injection (i.c.v.) induces an increase in BDNF and $p$-ERK1 in the dentate gyrus}

Analysis of homogenised tissue from the dentate gyrus collected 30 min post-injection showed an increase in BDNF concentration in the dentate gyrus of the BDNF-treated group compared to CytCtreated controls (Fig. 6a, $p=0.028$, Student's t-test), Western blot analysis revealed a significant increase in the phosphorylation of ERK1 ( $\mathrm{p} 44$-ERK) in the dentate gyrus of BDNF rats compared to the control group (Fig. 6b, $p=0.028$, Student's $t$-test). No changes in p-ERK2, total ERK1 or total ERK2 were observed between groups.

\section{Discussion}

The results presented here demonstrate that one week of forced moderate exercise can improve spatial memory, as assessed using the object displacement task, in young, healthy adult rats, in agreement with previous findings in our laboratory [16]. Other reports in the literature suggest that in order for short-term exercise to enhance spatial learning, a deficit (insult, lesion, stress, ageing) must be present [16,25-27]. The object displacement task allows the cognitive load to be varied by altering training time. We have previously shown that sedentary rats can learn less challenging variants of this task $(3 \times 5 \mathrm{~min}$ and $2 \times 5$ min training trials), while they cannot learn the more challenging variant $(1 \times 5 \mathrm{~min}$ of training) used in the present study [16]. The ability to detect an enhancement in performance following exercise by avoiding a ceiling effect provided the rationale for choosing these protocols in the present study.

We hypothesised that the observed improvement in spatial memory is linked with the activation of BDNF-stimulated intracellular signalling pathways. Many studies have reported an increase in expression of BDNF in the dentate gyrus with exercise in rats [5,6,28-31], and we have replicated this finding. However, it is likely that the activity-dependent release of BDNF, rather than its expression, is of greater relevance to short-term plasticity. In the present study, under constitutive conditions, no difference was observed in the amount of BDNF released from slices of dentate gyrus in any group. Upon stimulation with $50 \mathrm{mM} \mathrm{KCl,} \mathrm{BDNF} \mathrm{release}$ was significantly increased in the exercised group compared with the control naive group, indicating, for the first time, that exercise may enhance activity-dependent release of BDNF from the cells of the dentate gyrus during learning. Several mechanisms could underlie this finding. It is possible that exercise could simply be causing an increase in the amount of BDNF readily available for release. This is supported by the increase in BDNF mRNA observed in the dentate gyrus with exercise, and by previous experiments in our laboratory showing an increase in BDNF protein in the dentate gyrus with exercise [16]. BDNF is the only neurotrophin that 
a)

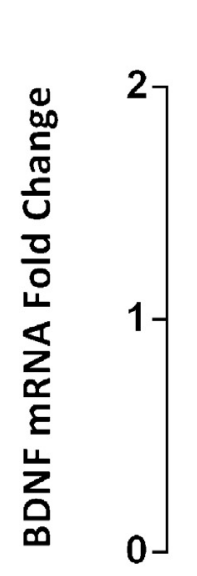

b)

$\star$

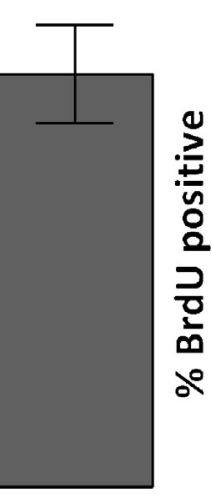

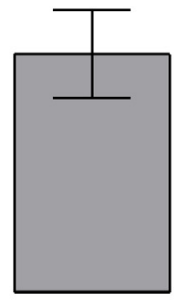

Sed

Ex

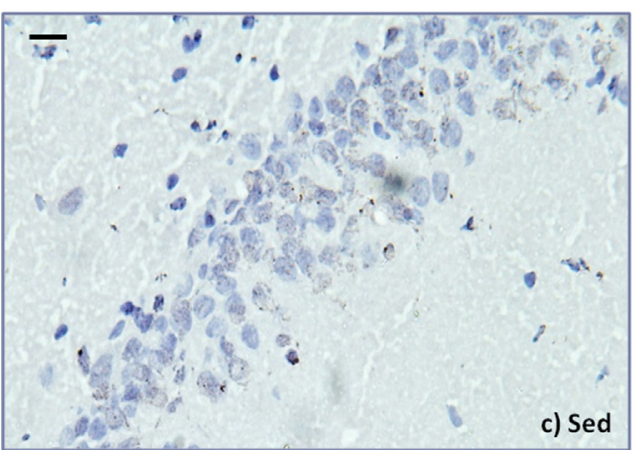

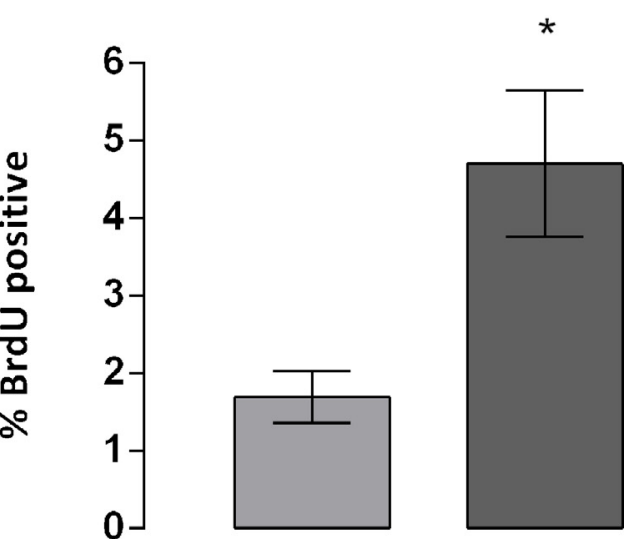

Sed
Ex

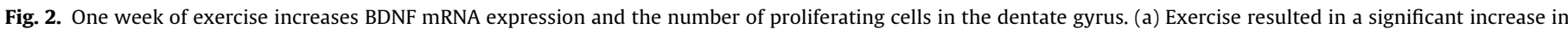

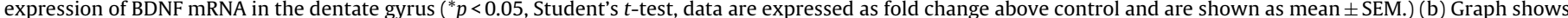

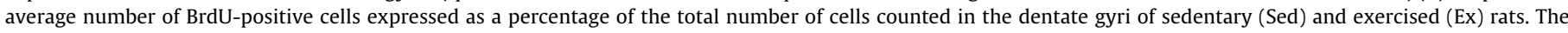

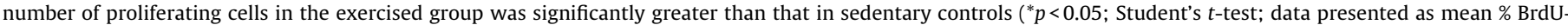

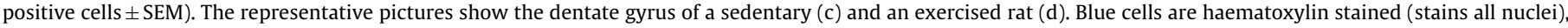
while brown cells are BrdU-positive (DAB chromagen stained). Scale bar $=200 \mu \mathrm{M}$.

is preferentially targeted for regulated secretion [32] as opposed to constitutive secretion, where the protein is packaged into synaptic vesicles that automatically exocytose once they reach the plasma membrane. The mechanisms by which exercise causes an increase in BDNF in the dentate gyrus are still unknown and merit further investigation. It is also possible that exercise could be causing an increase in the number and activity of synaptic vesicle proteins (such as synapsin-1) in the dentate gyrus. This could also be an indirect effect of increasing BDNF, since there is evidence that BDNF-stimulated intracellular signalling can affect synaptic vesicle protein activity [33]. Finally, since the tissue prepared from the dentate gyrus contains both neurons and glia, it is also possible that exercise is increasing the activity-dependent release of BDNF from glia. For example, astrocytes have been shown to take up pro-BDNF from the extracellular space and release it upon stimulation [34]. However, since most of the BDNF (90\%) present in the brain is in
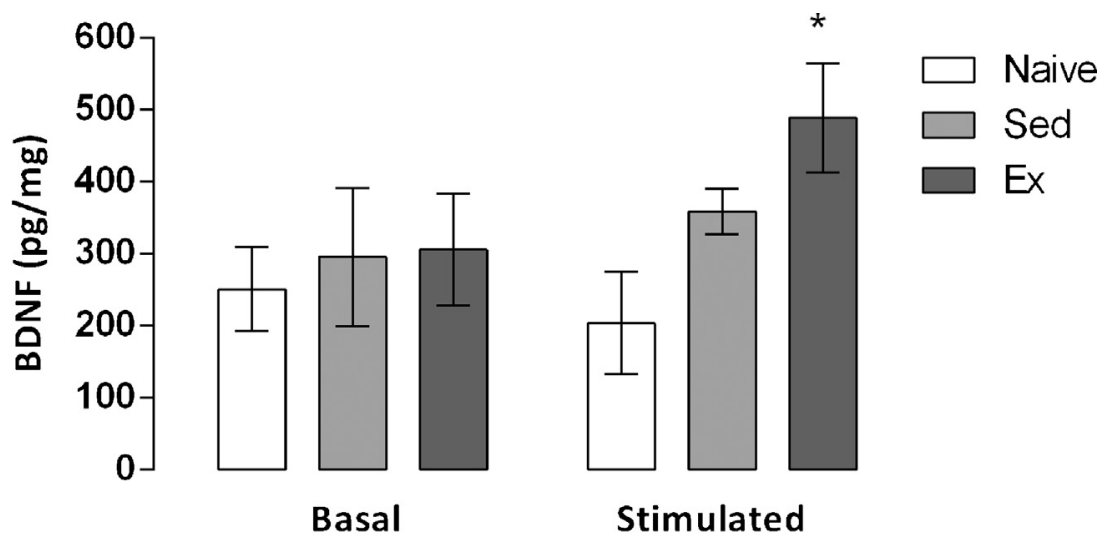

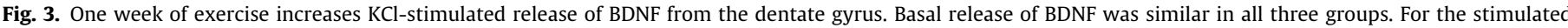

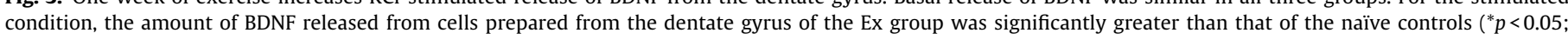
one-way ANOVA with Post hoc Tukey comparison; data are shown as mean BDNF pg/mg protein \pm SEM). 
a)

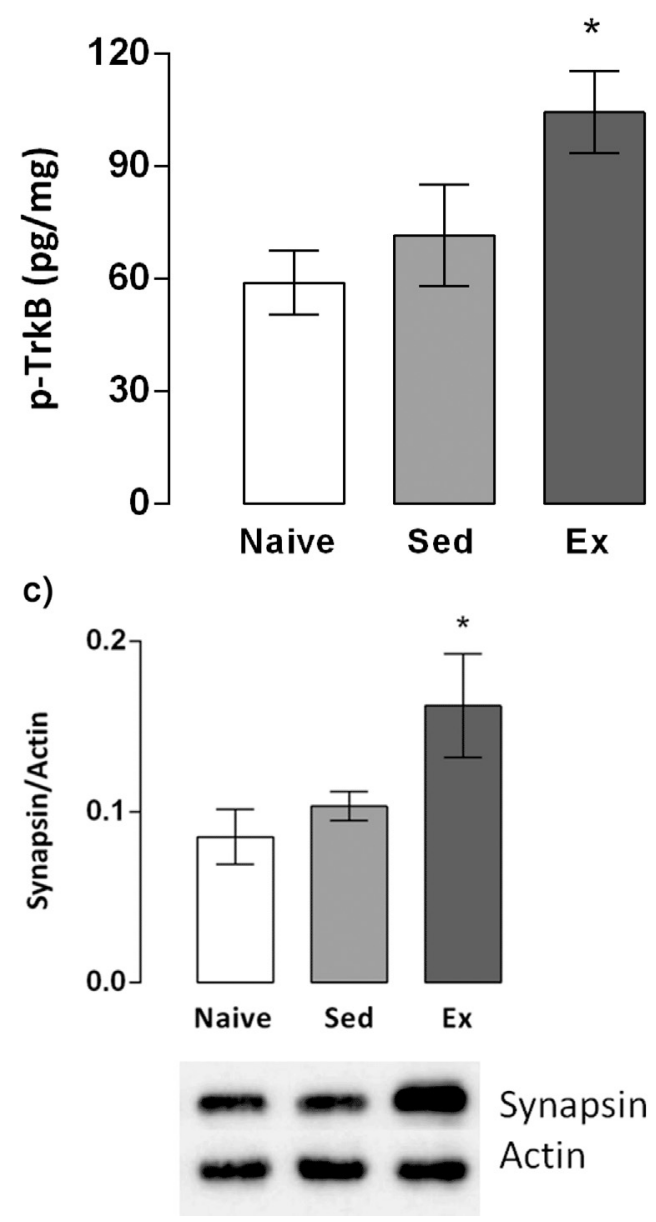

b)

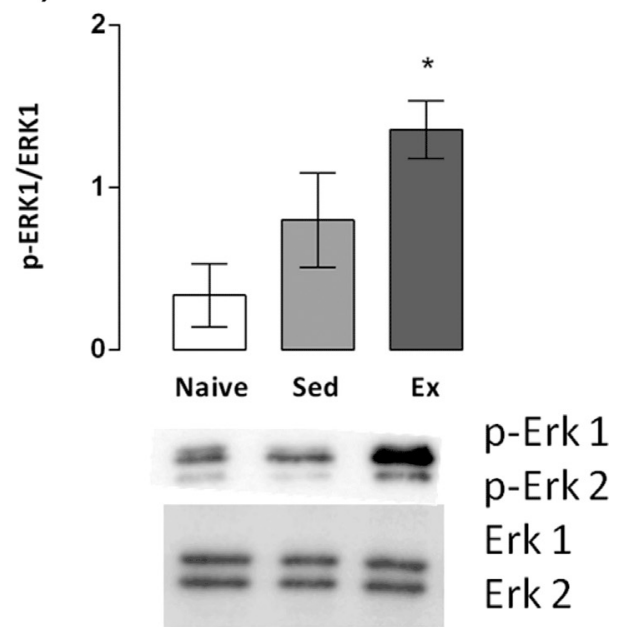

Actin

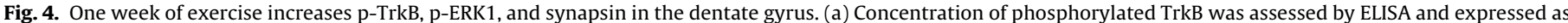

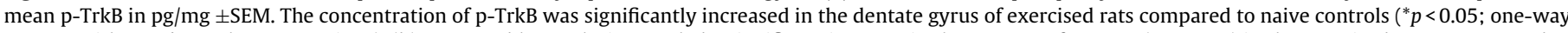

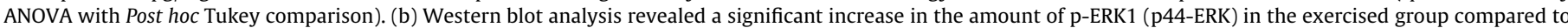

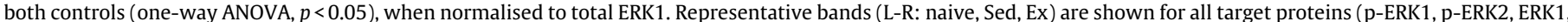

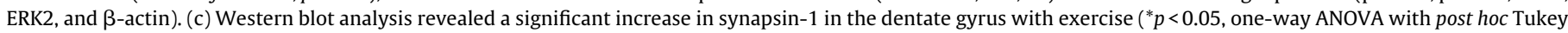

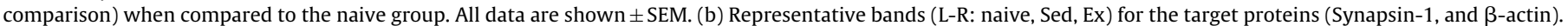

the mature form [35], it is unlikely that release of pro-BDNF from astrocytes contributes substantially to the BDNF release measured in the samples.

Upon release into the synaptic cleft, BDNF binds with high affinity to TrkB receptors, causing receptor dimerisation and autophosphorylation, and activation of specific signalling cascades including the ERK pathway [36,37]. In the adult mammalian brain, the highest levels of TrkB are expressed on granule cells of the dentate gyrus and pyramidal cells of the CA3 [38]. TrkB expression is also highest in association with glutamatergic synapses, the distribution pattern of which colocalises with BDNF expression. In the present study, we found an increase in the amount of phosphorylated TrkB protein in the dentate gyrus of exercised rats. This increase was significant only when compared to the naive controls, and not to the Sed group. This suggests that training in the spatial task may have led to some activation of the TrkB receptor, albeit not as strongly as with exercise. Activation of the TrkB receptor by BDNF, and the subsequent activation of signalling cascades has been shown to occur with learning [37]. The data suggest that exercise, by increasing BDNF, stimulates the same pathways that are activated by learning, causing amplification of the downstream effects and consequent improvements in memory acquisition and consolidation.
To test this hypothesis, we assessed activation of the downstream signalling proteins ERK1 and ERK2, members of the mitogen-activated kinase (MAPK) superfamily, which are known to be activated by the binding of BDNF to TrkB. We found an increase in the phosphorylation of ERK1 in the dentate gyrus in the exercised group only, while no changes were observed in ERK2 activation, or in total ERK1 or ERK2. Since exercise has been shown to increase BDNF in the hippocampus, it is not surprising that BDNF-related signalling cascades would become activated in response to exercise. Evidence has shown that the activation of the ERK pathway is essential to learning and memory $[14,15,20]$ and necessary for the protein synthesis-dependent stage of LTP. Gene targets of ERK include transcription factors such as CREB and Zif268 and plasticityrelated proteins such as Arc [39]. ERK activation has also been shown to upregulate BDNF [40], indicating that BDNF can activate its own transcription via ERK.

In the present study, only the ERK1 isoform, and not ERK2, was significantly activated in the exercised group. There is some evidence that these two isoforms of ERK may be differentially activated in different conditions. For example, it has been reported that only ERK1, and not ERK2, is activated in the dentate gyrus following object recognition learning [41]. Since ERK1 has been shown to be activated in the dentate gyrus with simple exposure to objects 
a)

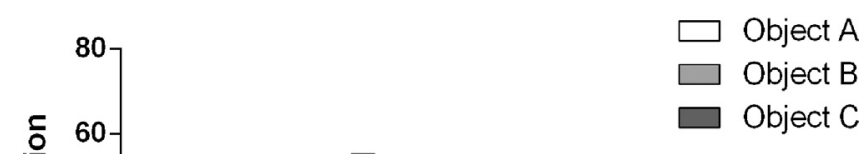

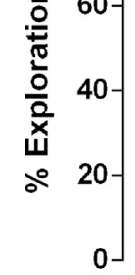

b)

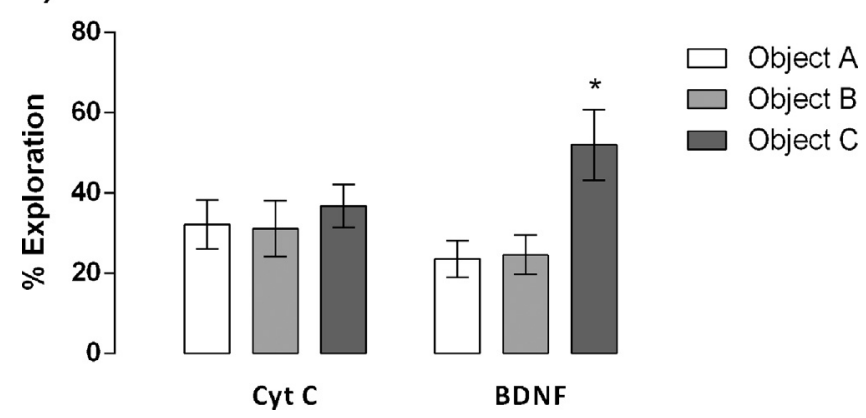

Fig. 5. Rats infused with BDNF (i.c.v.) display enhanced spatial learning. Neither of the groups showed a preference for any of the objects on training day (a). On the testing day (b), BDNF-treated rats preferentially explored the displaced object (object C; ${ }^{*} p<0.05$; one-way ANOVA with Post hoc Tukey comparisons) while the control group (CytC) did not. All data are shown as mean \% exploration time for each object \pm SEM.

(training) in an object recognition task [41], it is possible that the activation of ERK1 in the dentate gyrus could be learning-induced and not exercise-induced; a naive group was included in the study to control for any effects of exposure to the objects. The greatest phosphorylation of ERK1 was detected in the exercised group compared with either sedentary group, regardless of exposure to the objects. This provides evidence to suggest that the activation of ERK1 in the dentate gyrus we report here is exercise-specific.

We found an increase in the expression of synapsin- 1 in the dentate gyrus of exercised rats compared to Sed and naive controls. Synapsin- 1 is the most abundant phosphoprotein present in synaptic vesicle membranes of the mammalian central nervous system. It can be phosphorylated by several substrates, including ERK [33], and has been shown to be essential for the clustering and release of vesicles at the axon terminal [42], and hence synaptic transmission. Other studies have shown that synapsin-1 is upregulated in the hippocampus of adult rats with as little as three days of voluntary exercise [6], and here we report that forced moderate exercise induces a similar effect. In the study by Vaynman and colleagues, injecting the rats with the BDNF scavenger, TrkB-IgG, prevented the exercise-induced upregulation of synapsin-1, thus indicating that BDNF-TrkB signalling is required for the upregulation of synapsin-1 by exercise [6].

We report a significant increase in the number of BrdU-positive cells in the dentate gyrus after one week of exercise. This is in agreement with other studies that have reported cellular proliferation in the dentate gyrus of rats with exercise that is both forced and of short duration (less than three weeks $[43,44]$ ). Cell proliferation has been shown to increase after as little as three days of voluntary exercise in mice, becoming statistically significant only after 10 days [45]. However, there is some evidence that the stages of neurogenesis occur at a different rate in rats than in mice [46]. Our results demonstrate that cell division is increased in the dentate gyrus with a)
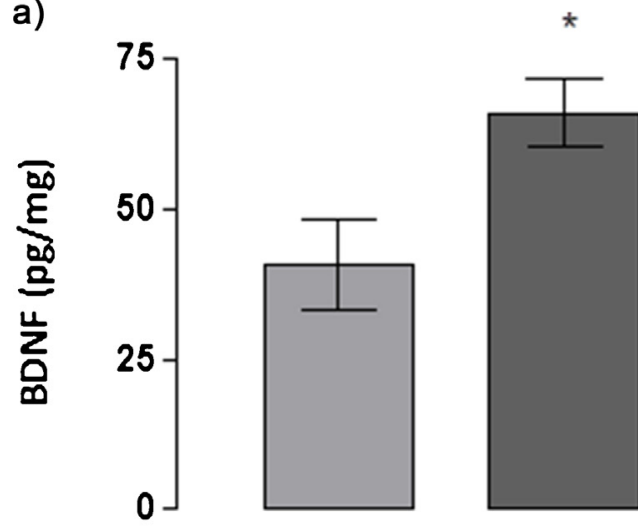

BDNF
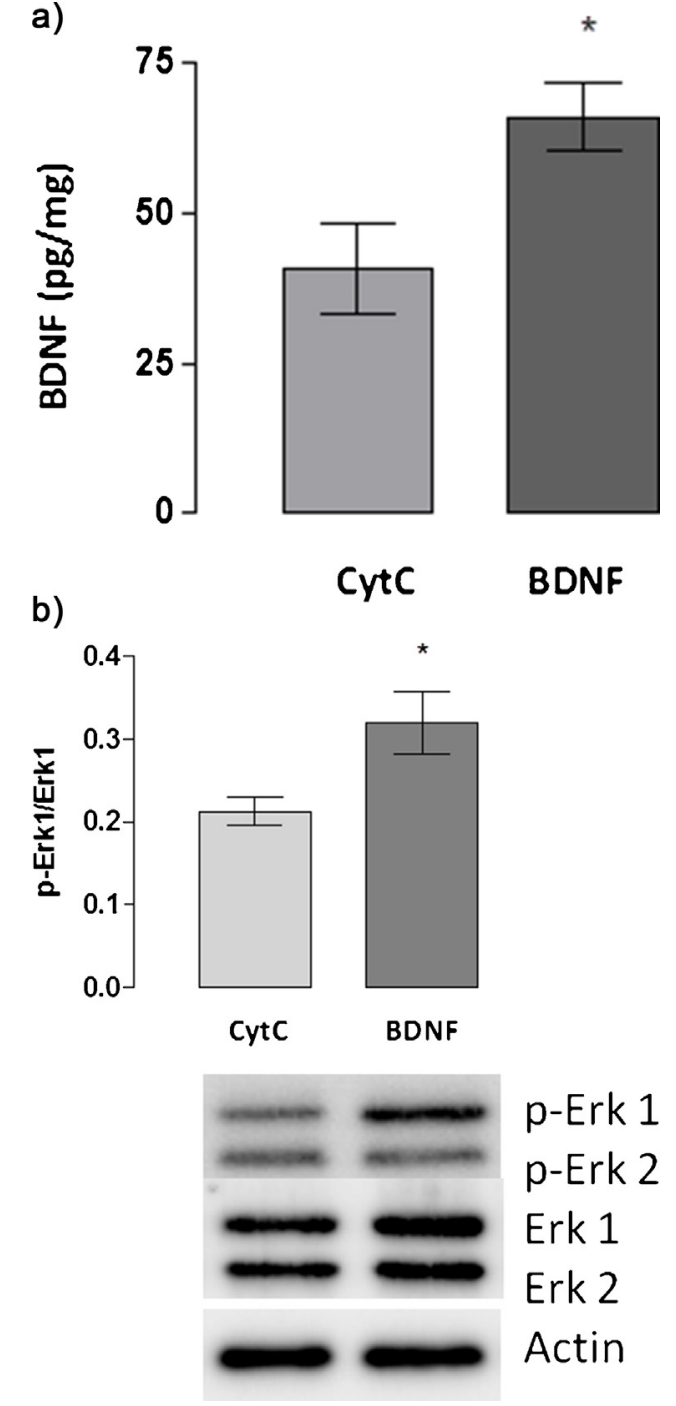

Fig. 6. A single BDNF injection (i.c.v.) induces an increase in the concentration of BDNF and pERK-1 in the dentate gyrus. (a) 30 min post-injection, there was a significant increase in BDNF concentration in the dentate gyrus of the BDNF-treated group when compared with CytC-treated controls $\left({ }^{*} p<0.05\right.$, Student's $t$-test). Data are presented as mean pg BDNF per mg of total protein \pm SEM. (b) Western blot analysis shows a significant increase in the expression of p-ERK1 (p44-ERK) in the dentate gyrus of BDNF-infused rats compared to CytC controls when normalised to total ERK1 (p44-ERK; * $\mathrm{p}<0.05$, Student's $t$-test). Representative bands (L-R: CytC, BDNF) for the target proteins p-ERK1, p-ERK2, ERK1, ERK2 and $\beta$-actin are shown. Data are presented as mean \pm SEM.

as little as one week of forced exercise; however, it is unlikely that this contributes to the observed improvements in spatial memory. First of all, cell division corresponds only to the first stage of neurogenesis, and proliferating cells include precursors that could give rise to different types of cells, including glia. Second, even if the proliferating cells are of neuronal phenotype, they would not have had sufficient time to mature or integrate into existing functional neuronal circuits [47] within 7 days. It is more likely that these cellular changes are involved in preparing the brain for future learning.

In an attempt to examine whether an exercise-induced increase in BDNF in the brain is sufficient to produce the observed effects on intracellular signalling and spatial learning, we assessed the efficacy of exogenous BDNF to mimic the increase caused by exercise. We report that a single intracerebroventricular (i.c.v.) injection of human recombinant BDNF improved performance in the spatial learning task. Exogenous BDNF administration has been shown previously to improve learning in non-spatial tasks, such as an 
inhibitory avoidance task [8] and a novel object recognition task [16]. One study reported some improvement in spatial learning in the Morris water maze with a single BDNF injection into the right hippocampus, however, in this case the authors were assessing memory retention, and the injection was administered post-training [48]. In addition, the concentration of exogenous BDNF administered in many published studies have been higher than the $10 \mathrm{ng}$ used in our laboratory $[8,48]$. In our experiment, the dose administered was based on the exercise-induced increase in BDNF observed in the dentate gyrus in previous experiments in our laboratory [16]. To the best of our knowledge, this is the first time that an exogenous BDNF injection at physiologically relevant concentrations has been reported to clearly improve memory in a spatial task. We observed an increase in BDNF protein in the contralateral dentate gyrus of rats that had received a single i.c.v. injection of recombinant BDNF. This result is noteworthy because it suggests that infused BDNF can diffuse from the left ventricle into hippocampal tissue, especially since it is unlikely that new BDNF is transcribed and translated within the indicated timeframe. Alternatively, BDNF could be diffusing to the hippocampus from other tissues as an indirect response to the i.c.v. injection, although we did not detect significant increases in BDNF in other areas (not shown). It has been suggested that the diffusion of exogenously administered BDNF is restricted, due in part to the large number of TrkB receptors that bind and rapidly internalise BDNF [49]. This could account for the difference in BDNF detected in the dentate gyrus compared to the amount infused. Nevertheless, these experiments support the hypothesis that an exercise-induced increase in BDNF in the dentate gyrus is sufficient to induce significant improvements in spatial memory.

Interestingly, a single i.c.v. injection of BDNF also resulted in significant activation of the ERK1 isoform in the dentate gyrus. Since no learning paradigms were performed in this experiment, we can conclude that in this case the activation of ERK1 is a direct consequence of the BDNF injection. The data suggest that an injection of BDNF into the ventricle can lead to the activation of BDNFstimulated signalling cascades in the dentate gyrus, presumably by diffusing into the brain parenchyma and activating TrkB receptors on granule cells, resulting in the subsequent activation of ERK1 in a manner that mimics the effect of exercise.

In conclusion, we propose that the temporal dynamics of adult hippocampal neurogenesis render it unlikely to mediate the cognitive benefits of one week of exercise. Although we observed increases in cell proliferation with one week of exercise, these new cells are more likely to be preparing the brain for future learning, which could be the function of many of the long-term effects of BDNF on the cytoarchitecture of the brain [50]. The observed cognitive improvement, if mediated by the consistent increases we observe in BDNF expression, are more likely to be due to the shortterm effects of this neurotrophin on synaptic transmission, which involve activation of the TrkB receptor, the subsequent activation of ERK1 and the upregulation and activation of synaptic vesicle proteins. This observed cognitive improvement can be mimicked by a single intracerebroventricular BDNF injection, which leads to the induction of signalling cascades and to the activation of kinases such as ERK1 that are crucial for plasticity and learning. Our data suggest that moderate forced exercise improves spatial learning by causing an increase in the activity-dependent release of BDNF in the dentate gyrus, and by subsequently inducing rapid cellular changes that facilitate synaptic transmission and plasticity.

\section{Acknowledgements}

Grant sponsors: Health Research Board of Ireland.

\section{References}

[1] Pang TY, Hannan AJ. Enhancement of cognitive function in models of brain disease through environmental enrichment and physical activity. Neuropharmacology 2013;64:515-28.

[2] Vaynman S, Gomez-Pinilla F. License to run: exercise impacts functional plasticity in the intact and injured central nervous system by using neurotrophins. Neurorehabil Neural Repair 2005;19:283-95.

[3] van Praag H, Kempermann G, Gage FH. Running increases cell proliferation and neurogenesis in the adult mouse dentate gyrus. Nat Neurosci 1999:2:266-70.

[4] Gomez-Pinilla F, Vaynman S, Ying Z. Brain-derived neurotrophic factor functions as a metabotrophin to mediate the effects of exercise on cognition. Eur J Neurosci 2008;28:2278-87

[5] Farmer J, Zhao X, van Praag H, Wodtke K, Gage FH, Christie BR. Effects of voluntary exercise on synaptic plasticity and gene expression in the dentate gyrus of adult male Sprague-Dawley rats in vivo. Neuroscience 2004;124:71-9.

[6] Vaynman S, Ying Z, Gomez-Pinilla F. Hippocampal BDNF mediates the efficacy of exercise on synaptic plasticity and cognition. Eur J Neurosci 2004;20:2580-90.

[7] Bekinschtein P, Oomen CA, Saksida LM, Bussey TJ. Effects of environmental enrichment and voluntary exercise on neurogenesis, learning and memory, and pattern separation: BDNF as a critical variable. Semin Cell Dev Biol 2011;22:536-42.

[8] Alonso M, Bekinschtein P, Cammarota M, Vianna MR, Izquierdo I, Medina JH. Endogenous BDNF is required for long-term memory formation in the rat parietal cortex. Learn Mem 2005;12:504-10.

[9] Bekinschtein P, Cammarota M, Katche C, Slipczuk L, Rossato JI, Goldin A, et al. BDNF is essential to promote persistence of long-term memory storage. Proc Natl Acad Sci U S A 2008;105:2711-6.

[10] Hall J, Thomas KL, Everitt BJ. Rapid and selective induction of BDNF expression in the hippocampus during contextual learning. Nat Neurosci 2000;3:533-5.

[11] Bramham CR, Messaoudi E. BDNF function in adult synaptic plasticity: the synaptic consolidation hypothesis. Prog Neurobiol 2005;76:99-125.

[12] Cunha C, Brambilla R, Thomas KL. A simple role for BDNF in learning and memory? Front Mol Neurosci 2010;3:1.

[13] Sweatt JD. The neuronal MAP kinase cascade: a biochemical signal integration system subserving synaptic plasticity and memory. J Neurochem 2001;76:1-10.

[14] Davis S, Laroche S. Mitogen-activated protein kinase/extracellular regulated kinase signalling and memory stabilization: a review. Genes Brain Behav 2006;5(Suppl 2):61-72.

[15] Peng S, Zhang Y, Zhang J, Wang H, Ren B. ERK in learning and memory: a review of recent research. Int J Mol Sci 2010;11:222-32.

[16] Griffin EW, Bechara RG, Birch AM, Kelly AM. Exercise enhances hippocampaldependent learning in the rat: evidence for a BDNF-related mechanism. Hippocampus 2009;19:973-80.

[17] Bechara RG, Kelly AM. Exercise improves object recognition memory and induces BDNF expression and cell proliferation in cognitively enriched rats. Behav Brain Res 2013.

[18] van Praag H. Neurogenesis and exercise: past and future directions. Neuromolecular Med 2008;10:128-40.

[19] Paxinos G, Watson C. The Rat Brain in Stereotactic Coordinates 2004.

[20] Margoliash E. Primary structure and evolution of cytochrome C. Proc Natl Acad Sci U S A 1963;50:672-9.

[21] Gooney M, Messaoudi E, Maher FO, Bramham CR, Lynch MA. BDNF-induced LTP in dentate gyrus is impaired with age: analysis of changes in cell signaling events. Neurobiol Aging 2004;25:1323-31.

[22] Messaoudi E, Ying SW, Kanhema T, Croll SD, Bramham CR. Brain-derived neurotrophic factor triggers transcription-dependent, late phase long-term potentiation in vivo. J Neurosci 2002;22:7453-61.

[23] Haan EA, Bowen DM. Protection of neocortical tissue prisms from freeze-thaw injury by dimethyl sulphoxide. J Neurochem 1981;37:243-6.

[24] Bradford MM. A rapid and sensitive method for the quantitation of microgram quantities of protein utilizing the principle of protein-dye binding. Anal Biochem 1976;72:248-54.

[25] Gobbo OL, O'Mara SM. Exercise, but not environmental enrichment, improves learning after kainic acid-induced hippocampal neurodegeneration in association with an increase in brain-derived neurotrophic factor. Behav Brain Res 2005; 159:21-6.

[26] Christie BR, Swann SE, Fox CJ, Froc D, Lieblich SE, Redila V, et al. Voluntary exercise rescues deficits in spatial memory and long-term potentiation in prenatal ethanol-exposed male rats. Eur J Neurosci 2005;21:1719-26.

[27] Cotman CW, Berchtold NC, Christie LA. Exercise builds brain health: key roles of growth factor cascades and inflammation. Trends Neurosci 2007;30:464-72.

[28] Soya H, Nakamura T, Deocaris CC, Kimpara A, Iimura M, Fujikawa T, et al. BDNF induction with mild exercise in the rat hippocampus. Biochem Biophys Res Commun 2007:358:961-7.

[29] Groves-Chapman JL, Murray PS, Stevens KL, Monroe DC, Koch LG, Britton SL, et al. Changes in mRNA levels for brain-derived neurotrophic factor after wheel running in rats selectively bred for high- and low-aerobic capacity. Brain Res 2011;1425:90-7

[30] Russo-Neustadt AA, Beard RC, Huang YM, Cotman CW. Physical activity and antidepressant treatment potentiate the expression of specific brainderived neurotrophic factor transcripts in the rat hippocampus. Neuroscience 2000;101:305-12. 
G Model

BBR-8593; No. of Pages 10

10

R.G. Bechara et al. / Behavioural Brain Research $x x x$ (2013) $x x x-x x x$

[31] Neeper SA, Gomez-Pinilla F, Choi J, Cotman CW. Physical activity increases mRA for brain-derived neurotrophic factor and nerve growth factor in rat brain. Brain Res 1996;726:49-56.

[32] Thomas K, Davies A. Neurotrophins: a ticket to ride for BDNF. Gur Biol 2005; 15:R262-4.

[33] Jovanovic JN, Czernik AJ, Fienberg AA, Greengard P, Sihra TS. Synapsins as meditors of BDNF-enhanced neurotransmitter release. Nat Neurosci 2000;3:323-9.

[34] Bergami M, Santi S, Formaggio E, Cagnoli C, Verderio C, Blum R, et al. Uptake and recycling of pro-BDNF for transmitter-induced secretion by cortical astrocytes. J Cell Biol 2008;183:213-21.

[35] Matsumoto T, Rauskolb S, Polack M, Klose J, Kolbeck R, Korte M, et al. Biosynthesis and processing of endogenous BDNF: CNS neurons store and secrete BDNF, not pro-BDNF. Nat Neurosci 2008;11:131-3.

[36] Huang EJ, Reichardt LF. Trk receptors: roles in neuronal signal transduction. Anu Rev Biochem 2003;72:609-42.

[37] Minichiello L. TrkB signalling pathways in LTP and learning. Nat Rev Neurosci 2009;10:850-60.

[38] Mure MG, Man Q, Raisman-Vozari R. Brain-derived neurotrophic factor in the control human brain, and in Alzheimer's disease and Parkinson's disease. Prog Neurobiol 2001;63:71-124.

[39] Zheng F, Luo Y, Wang H. Regulation of brain-derived neurotrophic factormediated transcription of the immediate early gene Arc by intracellular calcium and calmodulin. J Neurosci Res 2009;87:380-92.

[40] Saarelainen T, Vaittinen S, Castren E. trkB-receptor activation contributes to the kainate-induced increase in BDNF mRA synthesis. Cell Mol Neurobiol 2001;21:429-35.

[41] Kelly A, Laroche S, Davis S. Activation of mitogen-activated protein kinase/extracellular signal-regulated kinase in hippocampal circuitry is required for consolidation and reconsolidation of recognition memory. J Newrosci 2003;23:5354-60.

[42] Shupliakov O, Haucke V, Pechstein A. How synapsin I may cluster synaptic vesicles. Semin Cell Devi Biol 2011;22:393-9.

[43] Kim SH, Kim HB, Jung MH, Rim BV, Kim YJ, Kim YP, et al. Treadmill exercise increases cell proliferation without altering of apoptosis in dentate gyrus of Sprague-Dawley rats. Life Sci 2002;71:1331-40.

[44] Ida M, Ishido M, Kami K, Masuhara M. Effects of chronic treadmill running on neurogenesis in the dentate gyrus of the hippocampus of adult rat. Brain Res 2006;1104:64-72.

[45] Van der Borght K, Kobor-Nyakas DE, Klauke K, Eggen BJ, Nyakas C, Van der Zee EA, et al. Physical exercise leads to rapid adaptations in hippocampal vasculature: temporal dynamics and relationship to cell proliferation and newrogenesis. Hippocampus 2009;19:928-36.

[46] Snyder JS, Chou JS, Clifford MA, Jeurling SI, Hurley P, Brown A, et al. Adult-born hippocampal neurons are more numerous, faster maturing, and more involved in behavior in rats than in mice. J Neurosci 2009;29:14484-95.

[47] Ehninger D, Kempermann G. Neurogenesis in the adult hippocampus. Cell Thissue Res 2008;331:243-50.

[48] Cirulli F, Berry A, Chiarotti F, Alleva E. Intrahippocampal administration of BDNF in adult rats affects short-term behavioral plasticity in the Morris water maze and performance in the elevated plus-maze. Hippocampus 2004;14:802-7.

[49] Yarn Q, Rosenfeld RD, Matheson CR, Hawkins N, Lopez OT, Bennett L, et al. Expression of brain-derived neurotrophic factor protein in the adult rat central nervous system. Neuroscience 1997;78:431-48.

[50] Bekinschtein P, Cammarota M, Izquierdo I, Medina JH. BDNF and memory formation and storage. Neuroscientist 2008;14:147-56.

Please cite this article in press as: Bechara RG, et al. BDNF-stimulated intracellular signalling mechanisms underlie exercise-induced improvement in spatial memory in the male Wistar rat. Behav Brain Res (2013), http://dx.doi.org/10.1016/j.bbr.2013.11.015 